\title{
Modeling of polarization SAR system
}

\author{
D V Karasev', A N Leukhin ${ }^{2}$, A A Voronin ${ }^{2}$ and V I Bezrodny ${ }^{2}$ \\ ${ }^{1}$ Volga State University of Technology, Lenin Sq. 3, Yoshkar-Ola, Russia, \\ ${ }^{2} 424000$ Mari State University, Lenin Sq. 1, Yoshkar-Ola, Russia 424000
}

\begin{abstract}
We have implemented a combined mathematical model for polarization SAR. It allows simulating reflective objects on the background of the earth. We define the characteristics of underlying surface as an approximation of real experimental data. Value of backscattered field dependent on the angle of incidence. We get polarization characteristics of metal objects from the geometry configuration. It is based on the methods of physical optics and the physical theory of diffraction.
\end{abstract}

\section{Introduction}

Mathematical modeling plays important role in the creation of Synthetic Aperture Radar (SAR). It allows investigating the influence of o bservation c onditions in a wide $\mathrm{r}$ ange of parameters. Moreover, it allows us to develop new algorithms in lack of experimental data.

A promising method of increasing the informativeness of radar images is polarimetric data. Polarimetric SAR gives us extra information about properties of the underlying surface. This information makes it possible to classify structures on the image even if objects have similar shape or brightness on the image. This classification finds its application both in the military and civilian industry[1].

Computational complexity and amount of data of the SAR imaging are quite big. Often it is very hard to create real-time processing possible. A lot more data required to simulate radio wave propagation. For each point in radio hologram, we should calculate RCS for every object and the underlying surface. Precise methods such as Finite Element Method (FEM) or Finite Difference Time Domain (FDTD) method unsuitable in this c ase. A pproximate m ethods needs ratio $\lambda>3 \cdot l$ while precise $\lambda>10 \cdot l$ where $l$ - length of the largest element of mesh.

In this paper, we discuss the raycast methods that take into account the polarization characteristics. Such methods do not reflect $t$ he e xact $\mathrm{s}$ olutions of $\mathrm{t}$ he $\mathrm{w}$ ave e quations but provide a good approximation.

\section{Decomposition of the scattering matrix}

There are several methods for the scattering matrix decomposition. They represents scattering matrix as a linear combination of the matrices. Each of them corresponding to the basic scattering mechanisms [2].

One such method is the representation of the scattering matrix as a sum of the Pauli matrices:

$$
S=\frac{a}{\sqrt{2}}\left[\begin{array}{ll}
1 & 0 \\
0 & 1
\end{array}\right]+\frac{b}{\sqrt{2}}\left[\begin{array}{cc}
1 & 0 \\
0 & -1
\end{array}\right]+\frac{c}{\sqrt{2}}\left[\begin{array}{ll}
0 & 1 \\
1 & 0
\end{array}\right]+\frac{d}{\sqrt{2}}\left[\begin{array}{cc}
0 & -\mathrm{i} \\
\mathrm{i} & 0
\end{array}\right]
$$


The first term corresponds to the single scattering without change of polarization. The second term corresponds to a double reflection in which one of the orthogonal components changes sign. The third term represents the scattering-on dihedral reflector oriented at an angle of 45 degrees to the vertical. When wave reflected from such a reflector, the polarization changes to an orthogonal one. In the case of backscattering, the Pauli basis will include only the first three matrices.

Another form of representation of the scattering matrix is the Krogager decomposition. This decomposition consists of three elements. First one is equivalent of scattering on a sphere. The second one represents a dihedral corner reflector. The third element represents helix. For the last two types, the matrices depend on the orientation angle $\theta$ of the reflector.

The scattering matrix for this expansion is as follows:

$$
S=k_{\mathrm{s}} \cdot\left[\begin{array}{ll}
1 & 0 \\
0 & 1
\end{array}\right]+k_{\mathrm{d}} \cdot\left[\begin{array}{cc}
\cos 2 \theta & \sin 2 \theta \\
\sin 2 \theta & -\cos 2 \theta
\end{array}\right]+k_{\mathrm{h}} \cdot \mathrm{e}^{\mp \mathrm{i} 2 \theta} \cdot\left[\begin{array}{cc}
1 & \pm \mathrm{i} \\
\pm \mathrm{i} & 1
\end{array}\right] .
$$

The coefficients $k_{\mathrm{s}}, k_{\mathrm{d}}$, and $k_{\mathrm{h}}$ determine the contribution of the corresponding scattering mechanisms.

Both Pauli and Krogager decomposition allow a visual assessment of the geometry. Each decomposition reflects the degree of heterogeneity of the surface. It may be useful to distinguish between natural and artificial objects.

Another method provide the analysis based on the coherence matrix $T$. Elements of this matrix are calculated by transformations of scattering matrix $S$.

$$
T=\frac{1}{2}\left[\begin{array}{ccc}
\left(S_{\mathrm{HH}}+S_{\mathrm{VV}}\right) \cdot\left(S_{\mathrm{HH}}+S_{\mathrm{VV}}\right)^{*} & \left(S_{\mathrm{HH}}+S_{\mathrm{VV}}\right) \cdot\left(S_{\mathrm{HH}}-S_{\mathrm{VV}}\right)^{*} & 2\left(S_{\mathrm{HH}}+S_{\mathrm{VV}}\right) S_{\mathrm{HV}}^{*} \\
\left(S_{\mathrm{HH}}-S_{\mathrm{VV}}\right) \cdot\left(S_{\mathrm{HH}}+S_{\mathrm{VV}}\right)^{*} & \left(S_{\mathrm{HH}}-S_{\mathrm{VV}}\right) \cdot\left(S_{\mathrm{HH}}-S_{\mathrm{VV}}\right)^{*} & 2\left(S_{\mathrm{HH}}-S_{\mathrm{VV}}\right) S_{\mathrm{HV}}^{*} \\
2 S_{\mathrm{HV}}\left(S_{\mathrm{HH}}+S_{\mathrm{VV}}\right)^{*} & 2 S_{\mathrm{HV}}\left(S_{\mathrm{HH}}+S_{\mathrm{VV}}\right)^{*} & 4 S_{\mathrm{HV}} S_{\mathrm{HV}}^{*}
\end{array}\right] .
$$

The coherence matrix has three positive eigenvalues $\lambda_{1}, \lambda_{2}, \lambda_{3}$. In [3] it was suggested to use the relation:

$$
P_{j}=\frac{\lambda_{j}}{\lambda_{1}+\lambda_{2}+\lambda_{3}}, \quad j=1,2,3
$$

with the use of which a parameter was introduced, called the scattering entropy

$$
H=-\sum_{j=1}^{3} P_{j} \log _{3} P_{j}
$$

Scattering entropy represents the degree of randomness of the scattering, its values lie between 0 and 1 . The value $H=0$ corresponds to the perfect single reflection mechanism, and the value $H=1-$ complete diffuse scattering.

Coherence matrix can be reduced to diagonal form by transformation

$$
\begin{gathered}
T=U \cdot\left[\begin{array}{ccc}
\lambda_{1} & 0 & 0 \\
0 & \lambda_{2} & 0 \\
0 & 0 & \lambda_{3}
\end{array}\right] \cdot U^{-1}, \\
U=\left[\begin{array}{ccc}
\cos \alpha_{1} & \cos \alpha_{2} & \cos \alpha_{3} \\
\sin \alpha_{1} \cos \beta_{1} \exp \left(\mathrm{i} \delta_{1}\right) & \sin \alpha_{2} \cos \beta_{2} \exp \left(\mathrm{i} \delta_{2}\right) & \sin \alpha_{3} \cos \beta_{3} \exp \left(\mathrm{i} \delta_{3}\right) \\
\sin \alpha_{1} \cos \beta_{1} \exp \left(\mathrm{i} \gamma_{1}\right) & \sin \alpha_{2} \cos \beta_{2} \exp \left(\mathrm{i} \gamma_{2}\right) & \sin \alpha_{3} \cos \beta_{3} \exp \left(\mathrm{i} \gamma_{3}\right)
\end{array}\right]
\end{gathered}
$$

Based on the matrix $U$, the average angle can be calculated 


$$
\alpha=\sum_{j=1}^{3} P_{j} \alpha_{j},
$$

which characterizes the dominant scattering mechanism. A value of $\alpha=0^{\circ}$ corresponds to isotropic scattering on the surface, $\alpha=45^{\circ}$ - dipole scattering, and the value $\alpha=90^{\circ}$ - the double reflection.

Thus, methods of analyzing the coherence matrix allow us to divide natural objects into clusters with different scattering mechanisms.

\section{Modeling of underlying surface SAR}

The dimensions of the underlying surface are usually much larger than objects dimensions. It means that the surface needs an own approach to calculate scattered field.

We use a polygonal model of the surface. Each polygon has finite dimensions and corresponds with large-scale irregularities as presented at figure 1. In this case, the size of the facets should be less than the resolving power of the radar [4].

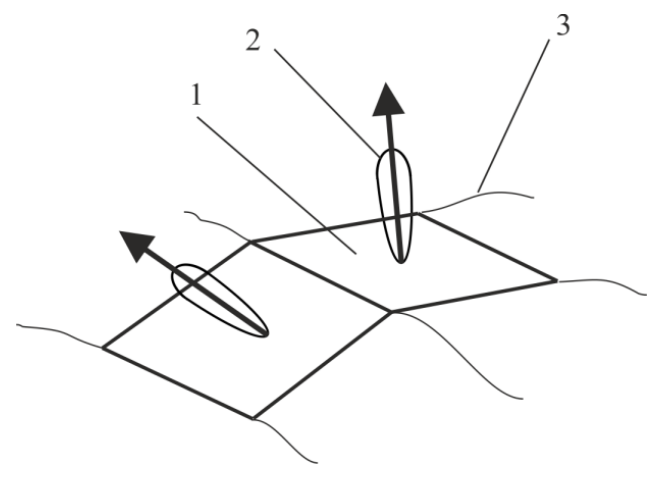

Figure 1. Faceted model of radar reflection by the earth's surface: 1 - facet; 2 - local backscattering diagram; 3 - surface.

The signal reflected from the surface is the sum of the signals from all the irradiated facets. The signal from individual facet has its own amplitude and its arbitrary phase. The relative position and backscattering diagram determine signal characteristics.

We have created a database of different t ypes of $\mathrm{r}$ eflecting ar eas. Ea ch ty pe ha s it s own material on the 3D model of a scene. Type of material connects the physical characteristics with the particular facet. An incident angle on backscattering diagram defines s pecific Ra dar Cross Section (RCS) value. Each material has three backscattering diagrams for each polarization. At this moment we do not take into account anisotropy of cross polarization. So we assume that $\mathrm{VH}$ polarization equals HV polarization.

For the backscattering diagram, we took the experimental data given in $[5,6,7]$. In these works, RCS value is a mean value of the particular material. Thus it is a statistical approach to simulate underlying surface properties. In range [0;90] degrees specific RCS is interpolated.

Examples of interpolated values of specific R CS in X-band a re represented in figure 2.

At angles close to the vertical scattering for most surfaces will be close to the mirror reflection and the highest values of specific RCS. At the angles close to the horizontal, the backscattering will be very small. At intermediate values of the slip angle, the specific RCS, expressed in $\mathrm{dB}$, varies with increasing slip angle according to a law close to linear. 


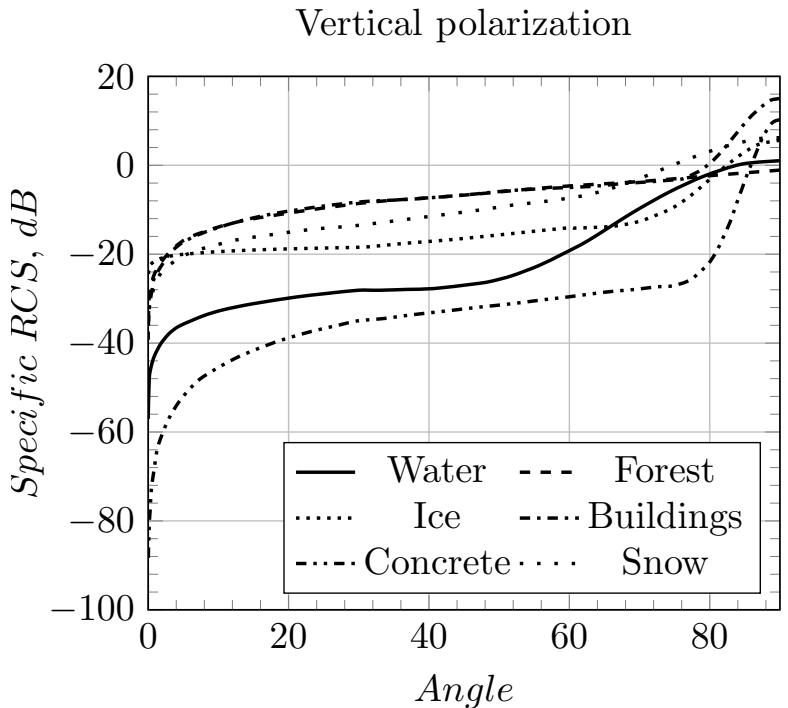

Horizontal polarization

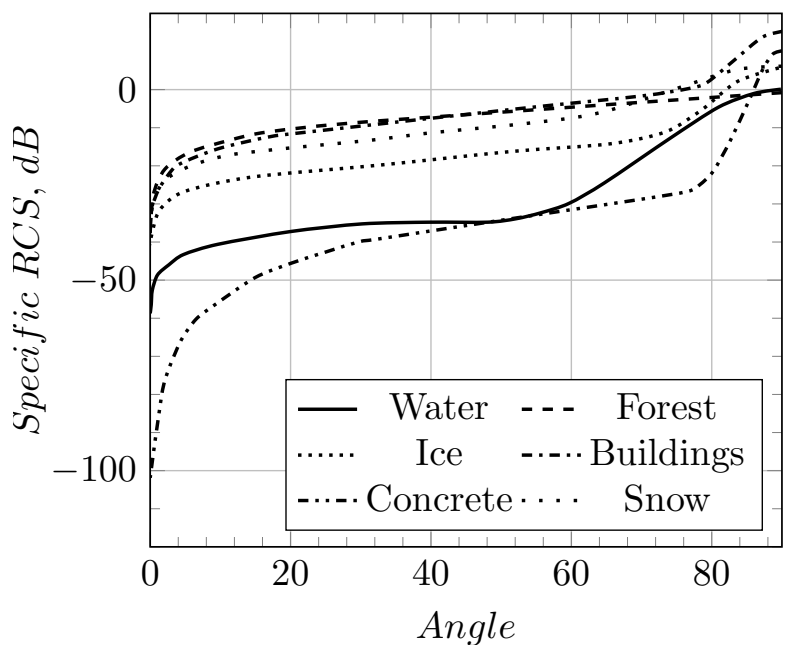

Cross polarization

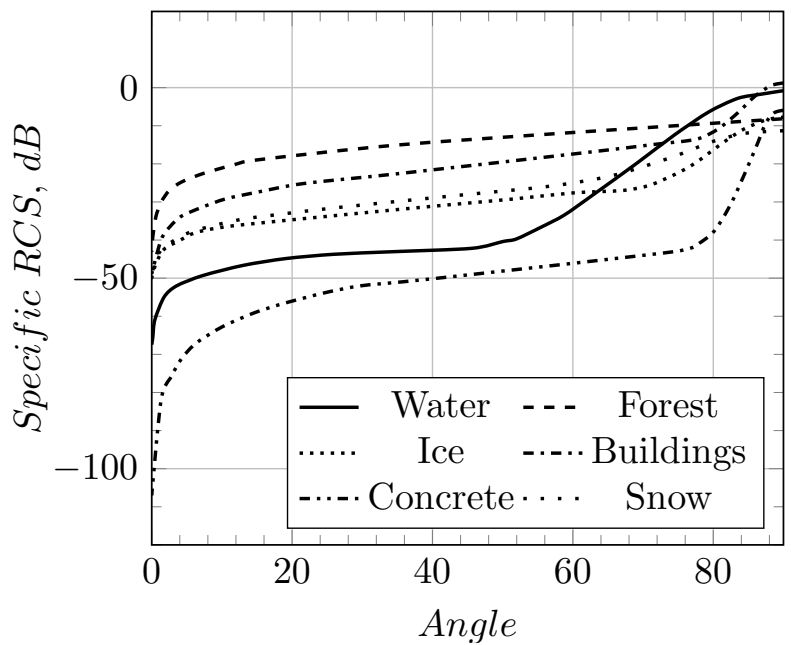

Vertical polarization

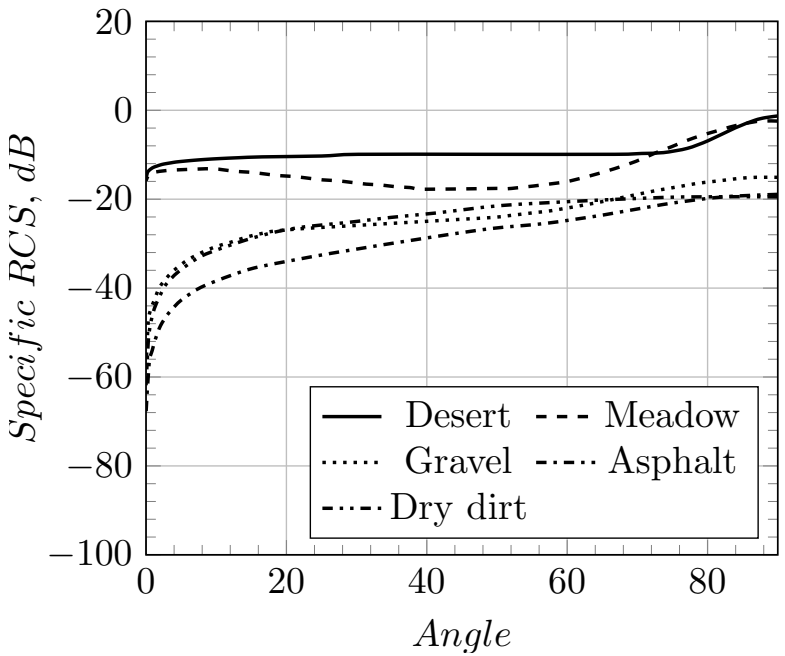

Horizontal polarization

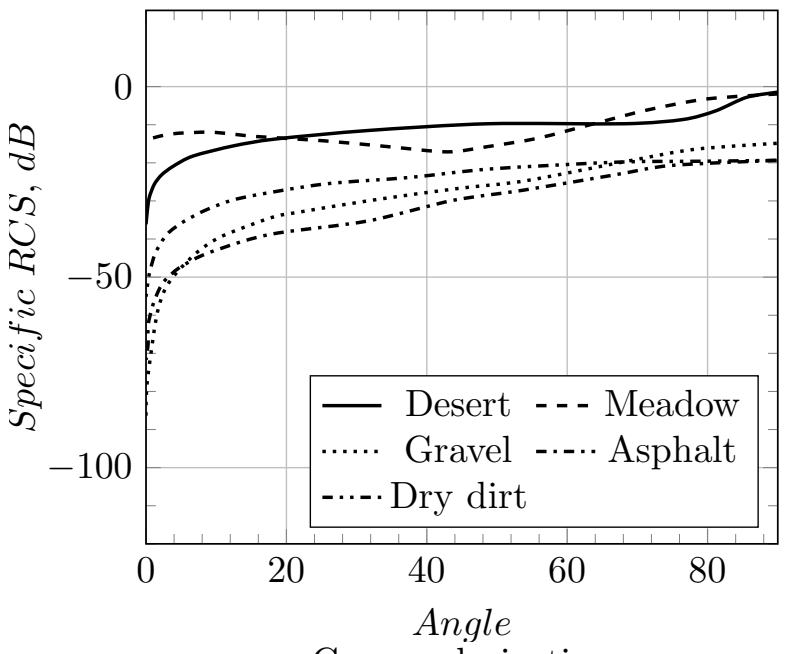

Cross polarization

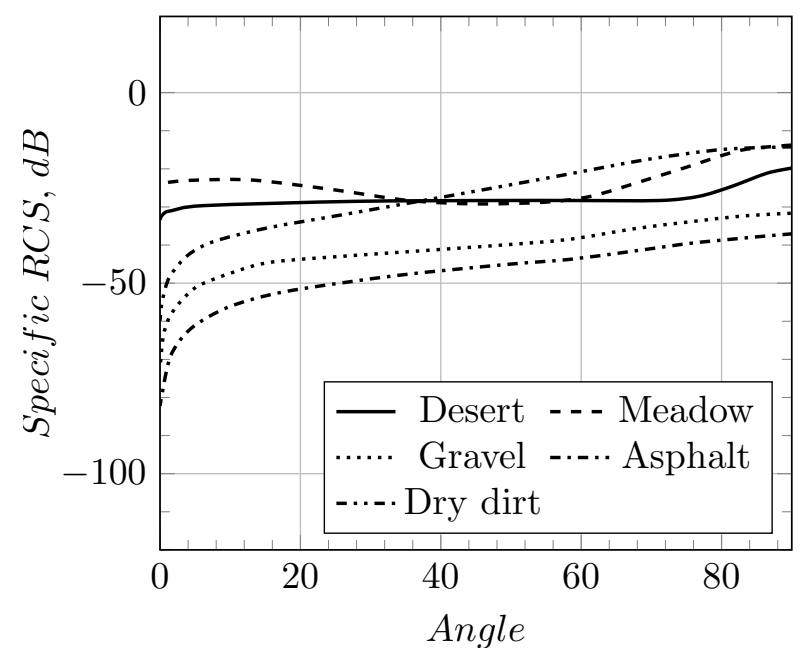

Figure 2. Dependence of specific RCS various surfaces of slip angle for different polarizations. 
Experiments using polarimetric SAR given in [8], show that for a phase difference between reflected waves with matched polarization and c ross-polarization has a uniform distribution in the interval $[0,2 \pi]$ for any distributed targets and, does not contain information about the target.

In contrast, the phase difference of $t$ he $r$ eflected ra dio wa ves on the ma tched polarizations depends both on the wavelength and the angle of incidence. Also, its influence has shape and dimensions, surface roughness, and material properties of the object.

Upon reflection from the r elatively s mooth $\mathrm{s}$ urfaces of $\mathrm{t}$ he $\mathrm{p}$ hase $\mathrm{d}$ ifference wi ll be cl ose to zero. In the case of double reflection, f or e xample $f$ rom $b$ uildings o $r t$ ree $t$ runks, $t$ he phase difference will be close to $180^{\circ}$. In scattering from an inhomogeneous medium, for example from vegetation, the phase difference can vary from 0 to $180^{\circ}$. In some cases, there may be joint effect of these scattering mechanisms.

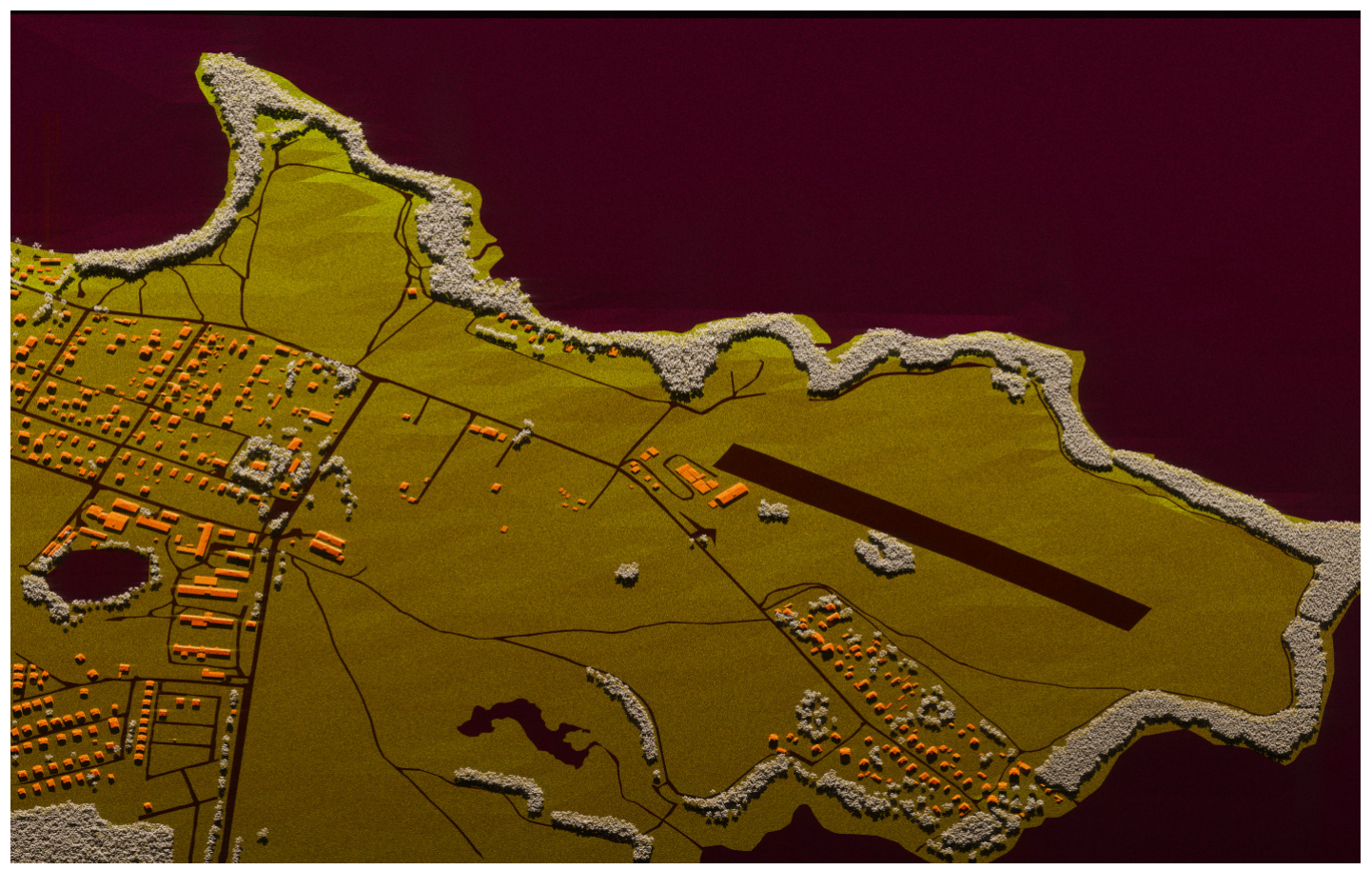

Figure 3. Radar image in pseudo colors.

Using the created model of radar signal reflections were simulated process of producing a radar image. Results are shown in figure 3. Different material types have their own pseudo colors: grass - yellow-green; houses — orange; trees - white; water — dark purple.

When generating radio holograms, the following model parameters were set:

- Flight altitude: $5 \mathrm{~km}$;

- Flight path: linear;

- Resolution: $30 \mathrm{~cm}$;

- Scanning angle: $45^{\circ}$;

- Modulation: phase;

- Carrier frequency: $10 \mathrm{GHz}$.

The simulated scene has the following dimensions: width $2700 \mathrm{~m}$; length of $2500 \mathrm{~m}$; height $40 \mathrm{~m}$. The average height of the houses is one floor. 
If a person tries to investigate each channel of the pseudo-color image as independent radio image, it will be hard to find a difference between VV and HH polarization. But anyway they do exist. The more obvious difference in comparison to cross-polarization. So, for the most comfortable representation combined image in pseudo-colors is needed. There are many ways to combine vertical, horizontal and cross polarization data, it may depend on the purpose of the SAR.

\section{Modeling of metal objects}

Often, of particular interest are metal objects created by man, especially for a military purpose. Due to the high conductivity, metal objects effectively reflect radio waves, and in th is case, the most interesting is the dependence of the RCS on the shape of the object, and also on the angle of the survey.

The model under discussion is a composition of different approximating methods based on the raycasting technique. The basic idea of composition proposed in a number of papers in which authors described relationships for the calculation of the scattered field taking into account the polarization and bounces of the rays $[9,10,11,12]$. Key methods described below.

William B. Gordon created far field a pproximation of t he $\mathrm{K}$ irchhoff fo rmula fo $\mathrm{r}$ a field scattered on a metal plate of an arbitrary form usually is given by some surface (double) integral. This double integral can be reduced to a linear integral estimated around the boundary. Moreover, if the boundary is a polygon, this integral can be reduced to a finite sum [13]. Polygon approximation of 3D shape allows us to use the fastest way to calculate scattered field.

Ufimtsev Petr Yakovlevich in his book [14] studies the diffraction of electromagnetic waves on bodies large in comparison with the wavelength. Approximate and strict methods are discussed. The results obtained give light to nature of such phenomena as Fresnel diffraction, shadow radiation, depolarization backscattering, the process of formation of edge waves, and so on.

Shyh-Kang Jeng proposes in his paper [15] a method to compute the near-field RCS and Doppler spectrum of a target when the distances to the antennas are comparable to the target size. By dealing with a small piece of the target surface at a time, the transmitting antenna, and the receiving antenna are in the far-field $\mathrm{z}$ one of $\mathrm{t}$ he $\mathrm{s}$ mall p iece of $\mathrm{t}$ he i nduced currents. The electromagnetic field p roduced by this s mall piece of induced c urrents can be written as a spherical wave. Sum up all spherical waves produced by every small piece of induced currents and we can obtain the total scattered field at the receiving antenna.

In figure $4 \mathrm{w}$ e u sed c olor $\mathrm{p}$ allet $\mathrm{w}$ ith s ome s caling c oefficients as fol lows: red - $\mathrm{HH}$ polarization, blue - VV polarization, green - VH $+\mathrm{HV}$ polarization.

When generating radio holograms, the following model parameters were set:

- Flight altitude: $5 \mathrm{~km}$;

- Flight path: linear;

- Resolution: $12.5 \mathrm{~cm}$;

- Scanning angle: $45^{\circ}$;

- Modulation: chirp;

- Carrier frequency: $10 \mathrm{GHz}$.

Each image oriented as follows: slope range from left to right, azimuth from top to bottom. Every object has the size of $2 \mathrm{~m}$ : width, height, length and diameter are equal to $2 \mathrm{~m}$.

It is clearly seen that the red and blue component dominate the majority of images. The brightest cross-polarization component is present in the image of the sphere and the cylinder. A significantly smaller amount of the green component is represented in the image of the cube. At the corner reflector, the bright cross-polarization component is present only in the center. In a square cross polarization plane missing almost completely. 


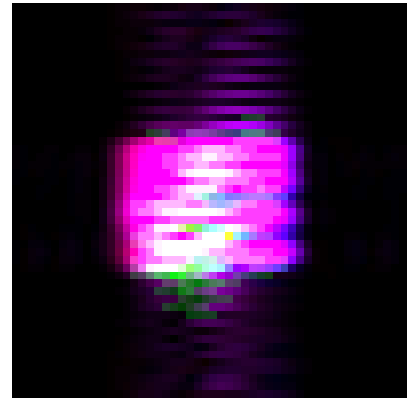

(a)

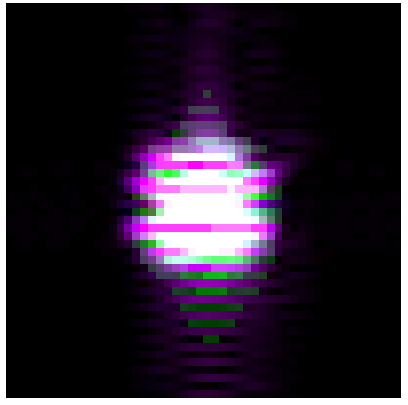

(b)

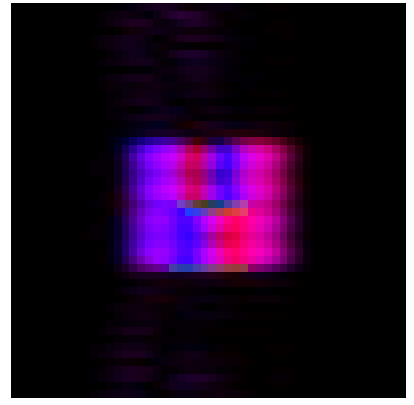

(c)

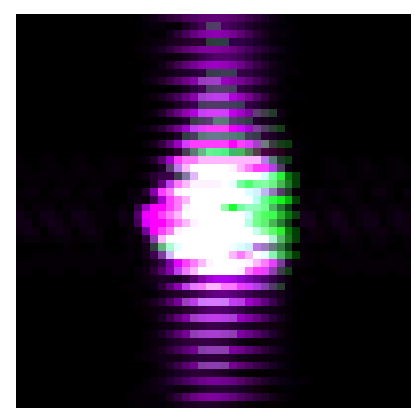

(d)

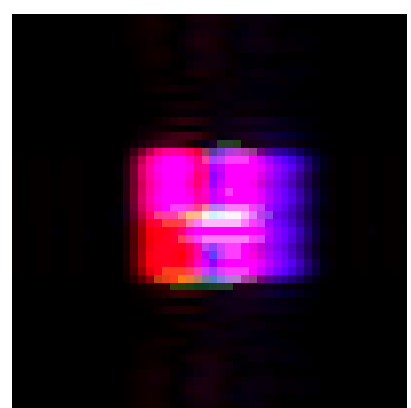

(e)

Figure 4. Radar image of the test objects in pseudo colors: (a) cube, (b) sphere, (c) plane, (d) cylinder, (e) corner reflector.

\section{Conclusions}

We implemented a mathematical model and SAR visualization using a combined technique. On the one hand, a statistical approach we used to model the underlying surface. This allows us to model large areas of view. On the other hand, for different $m$ etallic objects, we produce a more accurate calculation based on physical optics and the physical theory of diffraction. T he a pproach t o m odeling $d$ escribed in t his $\mathrm{p}$ aper a llows o btaining t est $\mathrm{d}$ ata for the development of image segmentation algorithms. Figure 4 shows the dependence of the polarization characteristics on the shape of the object. Such kind of information can be useful in tasks of target recognition.

\section{References}

[1] Afanasyev A A and Zamyatin A V 2017 Computer Optics 41(3) 431-440 DOI: 10.18287/2412-61792017-41-3-431-440

[2] Cloude S R and Pottier E 1996 IEEE transactions on geoscience and remote sensing 34 498-518

[3] Cloude S R and Pottier E 1997 Application of the $\mathrm{h} / \mathrm{a} / \alpha$ polarimetric decomposition theorem for land dassification Proc. SPIE 3120 132-143

[4] Baskakov A I, Zhutiaeva T S and Lukashenko Y I 2011 Locating methods of research objects and environments (Moscow: Academia) (in Russian)

[5] King C and Moore R K 1973 A survey of terrain radar backscatter coefficient measure program (Tech. Rep. The University of Kanzas, Center for Research Inc.) 2243

[6] Skolnik M 2008 Radar Handbook (New York: McGraw Hill)

[7] Katz I and Spetner L M 1960 Journal of Research of the National Bureau of Standards 64485

[8] Oh Y, Sarabandi K and Ulaby F T 2002 IEEE Transactions on Geoscience and Remote Sensing 40 1348-1355 
[9] Borzov A B, Suchkov V B and Sokolov A V 2004 Journal Of Radio Electronics (in Russian)

[10] Borzov A B, Suchkov V B, Shakhtarin B I and Sidorkina Y A 2014 Journal of Communications Technology and Electronics 59 1356-1368

[11] Akhiyarov V V, Borzov A B, Suchkov V, Shakhtarin B I and Sidorkina Y A 2015 Journal of Communications Technology and Electronics 60 1297-1304

[12] Suchkov V B 2013 Systems and means of communication, television, and broadcasting (in Russian)

[13] Gordon W B 1975 IEEE Transactions on Antennas and Propagation 23 590-592

[14] Ufimtsev P Y 2009 Theory of edge diffraction in electromagnetics (California: The Institution of Engineering and Technology)

[15] Jeng S K 1998 IEEE Transactions on Antennas and Propagation 46 551-558

\section{Acknowledgments}

The work is executed at financial support of the Ministry of Education and Science of the Russian Federation, project No. 2.2226.2017/Project Part and project No. 2.9140.2017/Basic Part. 